\title{
THE U.K. STARLINK COMPUTER NETWORK
}

David J. Carnochan

Dept. of Physics \& Astronomy, University College London

Six VAX-11/780 computers have been set up at different centres of astronomical research throughout the United Kingdom. These computers are solely for the use of astronomers and they are linked together using special telephone lines. They are proving to be a powerful tool, both in the reduction of astronomical images and in the subsequent data analysis.

StarLink is a network of six computers that are 'linked' together using telephone lines in a 'star' pattern; hence the name 'StarLink'. The six locations are: (1) RAL, the Rutherford \& Appleton Laboratories, (2) Cambridge University, (3) ROE, the Royal Observatory, Edinburgh, (4) Manchester University, (5) RGO, the Royal Greenwich Observatory, and (6) UCL, University College London. The computer at RAL is at the centre and the other sites form a five-pointed star.

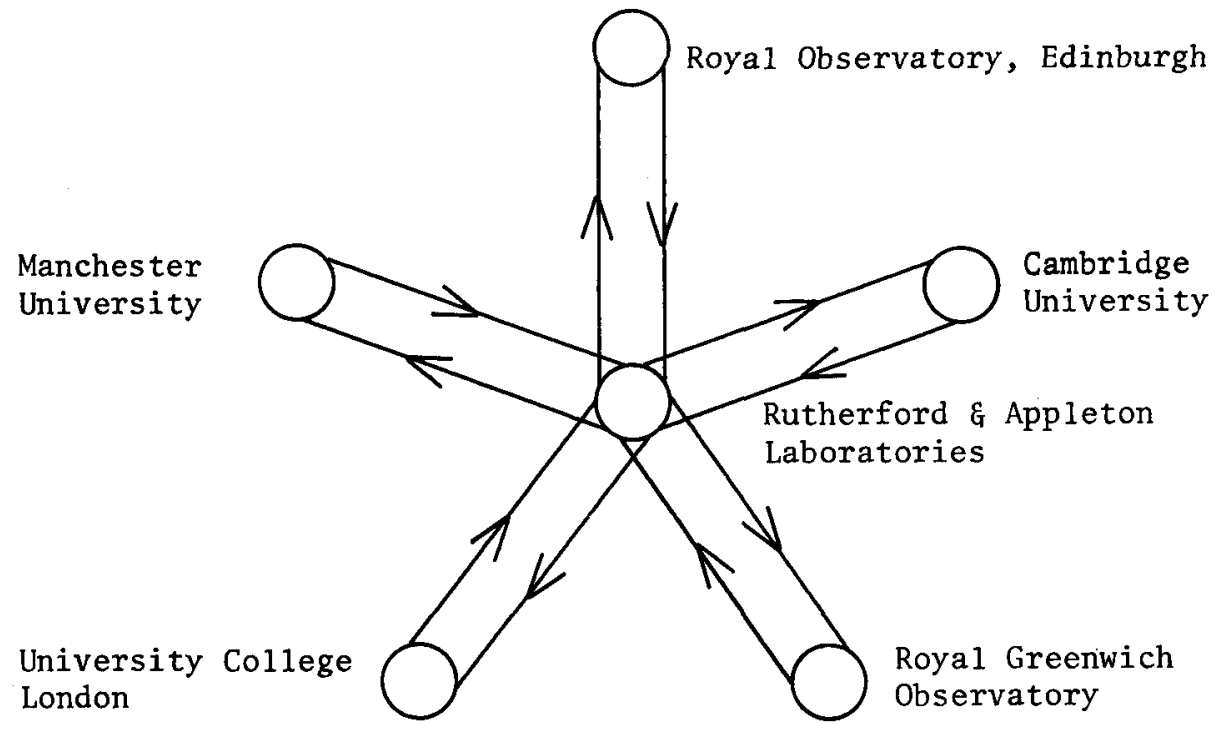

63

C. Jaschek and W. Heintz (eds.), Automated Data Retrieval in Astronomy, 63-65. Copyright $\odot 1982$ by D. Reidel Publishing Company. 
The transfer of data between sites has to go through the RAL node. Thus RAL can communicate directly with the other five computers, and vice-versa. But ROE can only communicate with RGO by following the path ROE - RAL RGO. The system used to link the computers together is DECnet which is a product of Digital Equipment Corporation (DEC) who make the VAX computers.

Simple linking operations whereby one site serves as a remote terminal are straightforward. An example of this is provided by a Manchester astronomer accessing the RGO computer (via RAL) and using an RGO program to draw finding-charts. However, more sophisticated applications are also possible. An astronomer at ROE can execute a UCL program for analysing IUE spectra, he can take an IUE image held at Cambridge and superpose over its spectrum a selection of spectral lines taken from a line list at RGO. DECnet is a relatively slow network and its use is only encouraged for the transfer of small amounts of data. A spectrum with 1000 data points is transmitted in a few seconds, but a $500 \times 500$ image takes many minutes.

Many modern astronomical experiments produce two-dimensional images that contain a very large number of data elements. These include photographic plates (which after scanning on a microdensitometer typically result in images of $100,000 \times 100,000$ pixels); IPCS, the Image Photon Counting System (which typically has images of $1,000 \times 1,000$ pixels) and, IUE, the International Uitraviolet Explorer satellite (where every image is $768 \times 768$ pixels). In order to handle these images, each StarLink site is provided with graphics terminals where the data can be displayed \& manipulated, both in black \& white and in colour. 'Hard-copy' devices are also available so that a permanent record of any image can be made.

Another important consideration of data reduction \& analysis is that every image tends to be 'special' demanding to be handled in its own unique way. Consequently, StarLink has been set up to run interactively. Although data can be reduced using 'black-box' programs in batch, most astronomers now prefer to manipulate the data themselves. On StarLink, the results are displayed back on the user's terminal as soon as the data is processed. The astronomer can react to the changed image immediately and adjust the flow of his analysis accordingly. StarLink provides the astronomer with the ability to do the analysis that he wants to do, rather than being limited to what a rigid system provides.

For example, consider the analysis of stellar spectra. For our first image we might be interested in identifying lines and require access to lists of spectral lines. With our second image we might find that the star has emission lines and need to switch over to a program that will fit P-Cygni profiles. For our third star we might be interested in interstellar effects and wish to difference its spectrum with a standard star in order to get at the extinction curve. And with our fourth image we might become a theoretician and not wish to process the image at all, but to 'process' instead a model-atmosphere \& line-profile program to get at a best fit model. All of these are possible with StarLink and many have already been implemented and are available now.

Of course, not every piece of analysis needs to use the StarLink network in order to access the other sites. Indeed each site has its own particular interests and the majority of work is carried out as if each computer were a stand-alone machine. RAL controls StarLink, provides much of the hardware \& software, and coordinates the different site activities. RAL also serves as the StarLink node for Oxford University where one area of interest is in reducing plates of clusters of galaxies. Edinburgh uses StarLink to analyse photographic 
plates that have been scanned using the 'Cosmos' microdensitometer, most of these plates are taken with the Anglo-Australian Schmidt telescope. Manchester processes data collected by the Jodrell Bank radio telescopes. RGO works in many areas including the three-dimensional images obtained with a Fabry-Perot interferometer used in conjunction with IPCS, typically for analysing of the velocity distributions in extended objects such as galaxies. UCL is primarily concerned with IUE images and with the IPCS observations of quasars. Cambridge, of course, does a bit of everything.

StarLink is not an isolated network of computers, and connections can be made into the general network that links together the majority of computers within the UK Science Research Council. However the special linking of identical machines is now lost and the StarLink computer then serves as a simple remote terminal.

For the future, the 'Universe' project is researching into the use of 'rings' for communicating large quantities of data between computers. Simple dial-up terminals transfer a few hundred bits per second, DECnet on StarLink can handle a few kilo-bits per second; rings offer the possibility of transmitting mega-bits per second. Rings are used to connect different computers that are physically very close to one another. Packets of data are sent from a machine's station into the ring. The packets pass through the stations of the other computers in a fixed direction around the ring and they leave when they arrive at the requested destination. One ring links the Cambridge University computers and another ring links the UCL computers. One of the stations in the ring can be a ground-station in which case the packet of data can be transmitted up to a satellite and back down into a different computer ring, perhaps half-way around the world. StarLink is involved in the Universe project and the UCL Vax will be used to help investigate the problems of moving bulk data between computers. 